\title{
The Immature Granulocyte Count Is a New Predictor of the 30-Day Mortality in Intracerebral Haemorrhage Patients: Preliminary Study
}

\author{
Cihan Bedel $^{1}$ Mustafa Korkut ${ }^{1}$ Fatih Selvi ${ }^{1}$ Ökkeş Zortuk ${ }^{1}$ \\ ${ }^{1}$ Department of Emergency Medicine, Health Science University, \\ Antalya Training and Research Hospital, Antalya, Turkey \\ Address for correspondence Cihan Bedel, MD, Health Science \\ University Antalya Training and Research Hospital, Kazım Karabekir \\ Street, Postal Zip Code: 07100, Muratpaşa, Antalya, Turkey \\ (e-mail: cihanbedel@hotmail.com).
}

Indian J Neurosurg 2021;10:114-120.

\begin{abstract}
Objectives Spontaneous intracerebral hemorrhage (ICH) accounts for 10 to $20 \%$ of all types of stroke and is associated with high mortality and morbidity rates. Neuroinflammation caused by intracerebral blood includes resident microglia activation, infiltration of systemic immune cells, and production of cytokines, chemokines, extracellular proteases, and reactive oxygen species. Despite several findings demonstrating that an immature granulocyte (IG) count can be a prognostic indicator as an inflammatory parameter in many diseases, no studies conducted on ICH patients are available in the literature. Therefore, in this study, we aimed to investigate the relationship between the 30-day mortality rate and the IG count obtained at the time of admission in $\mathrm{ICH}$ patients.

Methods Demographic characteristics and laboratory test results of patients, who were diagnosed with $\mathrm{ICH}$ and hospitalized accordingly upon arrival at the emergency ward in our tertiary care hospital in the period from January 2019 and December 2019, were recorded. The endpoint of the study was the examination of the relationship between the short-term mortality (within 30 days after hospitalization) and the IG count at admission.

Results Seventy patients, who met the inclusion criteria, were included in the study. Of these patients, 40 (57.1\%) were males and the mean age was $68.04 \pm 13.08$ years. Patients with poor prognosis had higher IG counts $(p=0.001)$. The 30 -day mortality rate was $33.3 \%(11 / 33)$ in the high IG count $(\geq 0.6)$ group and $5.4 \%(2 / 37)$ in the low IG

\section{Keywords}

- mortality

- immature granulocyte

- spontaneous intracerebral bleeding count $(<0.6)$ group $(p=0.004)$. In the regression analysis, we found out a significant relationship of the IG count to the 30-day mortality, with an odds ratio of 5.157(95\% $\mathrm{Cl}=0.914-29.087, p=0.029$ ).

Conclusion An IG count can be obtained from a simple full blood count, is easy to apply, does not result in extra costs, and is used as a marker to predict the 30-day prognosis.

published online January 6, 2021

DOI https://doi.org/

$10.1055 / \mathrm{s}-0040-1721627$ ISSN 2277-954X.

(c) 2021. Neurological Surgeons' Society of India.

This is an open access article published by Thieme under the terms of the Creative Commons Attribution-NonDerivative-NonCommercial-License, permitting copying and reproduction so long as the original work is given appropriate credit. Contents may not be used for commercial purposes, or adapted, remixed, transformed or built upon. (https://creativecommons.org/licenses/by-nc-nd/4.0/).

Thieme Medical and Scientific Publishers Pvt. Ltd. A-12, 2nd Floor Sector 2, Noida-201301 UP, India
\end{abstract}




\section{Introduction}

Spontaneous intracerebral hemorrhage (ICH) is a clinical manifestation of nontraumatic arterial or venous blood escape into the brain tissue. ICH is responsible for 10 to $20 \%$ of all stroke types and is associated with high mortality and morbidity. ${ }^{1}$ The severity of the clinical picture is associated with the volume of extravasated blood mass; the mass, extent, and the location of the haematoma; age, and Glasgow coma scale (GCS) scores. ${ }^{2}$ Inflammatory processes are increasingly recognized as important players in the pathophysiology of secondary brain injury after $\mathrm{ICH} .{ }^{3}$ Many studies have recently been reported supporting that inflammatory responses take a significant part in $\mathrm{ICH}^{4-6}$

Within this scope, the neutrophil count, the neutrophil/ lymphocyte ratio (NLR), the thrombocyte/lymphocyte ratio (PLR), and the levels of C-reactive protein (CRP) and interleukin-6 as inflammatory parameters have been proposed to predict the prognosis of $\mathrm{ICH}^{7}{ }^{7}$ Recent studies have reported that immature granulocytes (IG) detectable with automated blood cell analyzers in the peripheral blood indicate bone marrow activation in infection and sepsis. ${ }^{8}$ A high IG count is a robust prognostic factor in bacteremia, acute appendicitis, and complications of pancreatitis. ${ }^{9-11}$ However, to the best of our knowledge, no studies have examined the relationship between IG and prognosis in ICH patients previously. Therefore, in this study, we wished to investigate the relationship between the 30-day mortality and the IG count obtained at admission in ICH patients.

\section{Methods}

This retrospective observational study included patients, who were diagnosed and hospitalized with acute ICH upon admission at our tertiary emergency room in the period from January 2019 to December 2019. The diagnosis of ICH was made after obtaining the patient's history, making the neurological examination, and detecting the hemorrhagic lesion in the computed tomography (CT). Subtyping of ICH detected by CT (hypertensive bleeding, aneurysmal hemorrhage, amyloid angiopathy hemorrhage and microvascular hemorrhage) applied to our study population. The exclusion criteria of the study were defined as follows: having systemic inflammatory diseases, hematological diseases, malignant diseases, myocardial infarction (MI), heart failure (HF), evidence of active infection at the time of admission, recent major trauma, and acute poisoning; the use of cytotoxic chemotherapy causing bone marrow suppression, and missing laboratory and imaging findings. Age, gender, medical history, risk factors, vital signs, any surgical procedures, clinical laboratory test results within the first hour after emergency room admission, GCS scores, and CT imaging data were retrieved for all patients.

Of the laboratory parameters, glucose levels; white blood cell, neutrophil, lymphocyte, platelet; IG percentages and counts, and CRP levels were recorded. The neutrophil-to-lymphocyte ratio (NLR) and the platelet-to-lymphocyte ratio (PLR) were calculated. In the CT images; the volume and location (lobar, cerebellar, ganglial) of the bleeding and whether intraventricular hemorrhage (IVH) was present were identified and recorded. Confirmed 'ABC/2' or ' $\mathrm{ABC} / 3^{\prime}$ methods in previous studies were used to estimate the hematoma volume for "round and ellipsoid" or "irregular and uniquely shaped" hemorrhages, respectively. ${ }^{11,12}$ The study endpoint was to examine the relationship between short-term mortality (within 30 days after hospitalization) and the IG count measured at the time of admission.

The statistical analysis of all variables was performed by using SPSS version 23.0. Continuous variables were expressed as mean \pm standard deviation (SD). Frequency and percentage (\%) were used to define categorical data. Pearson's Chi-square test was used for the evaluation of categorical variables. For comparing the parameters between the good and poor outcome groups and the groups of patients with an IG count of $\geq 0.6$ and IG count of $<0.6$ as reference values, the Student $t$-test and the Mann-Whitney $U$ test were used for normally and non-normally distributed variables, respectively. A multiple logistic regression was applied to identify factors involved in the 30-day mortality. The optimum cutoff value of the IG count to predict the 30-day survival was analyzed with the receiver operating characteristic (ROC) analysis. In comparisons between groups in the study, a $p$ value of $<0.05$ was considered statistically significant.

\section{Results}

Seventy patients, who met the inclusion criteria, were included in the study. Of these patients, 40 (57.1\%) were males and the mean age was $68.04 \pm 13.08$ years. The mean white blood cell (WBC) count of the patients was $10.97 \pm 4.0410^{3} / \mathrm{mL}$, the mean NLR was $6.91 \pm 5.86$, and the mean IG count was $0.76 \pm$ 0.08 . The main clinical features of the patients are detailed in - Table 1. The patients were divided into two groups as the good outcome and poor outcome groups according to the 30-day mortality status. Of the study patients, 57 (81.4\%) were in the good outcome group and 13 (18.6\%) were in the poor outcome group. The patients in the poor outcome group had a higher ICH volume in the imaging tests $(76.98 \pm 20.49$ vs. $31.24 \pm$ 5.64). ICH was lobar at a higher percentage in the patients in the poor prognosis group. Of the laboratory parameters, the WBC count $(13.50 \pm 4.34$ vs. $10: 39 \pm 3.78 ; p=0.020)$, the neutrophil count ( $10.42 \pm 3.51$ vs. $7.72 \pm 3.67 ; p=0.011)$, NLR $(9.11 \pm$ 5.76 vs. $6.41 \pm 5.82 ; p=0.005$ ), and the IG count ( $1.24 \pm 0.66$ vs. $0.65 \pm 0.18 ; p=0.001$ ) were higher in the poor prognosis group compared with the good prognosis group (-Table 1).

Of the 70 patients, 37 had a low IG count $(<0.6)$ and the remaining 33 had a high IG count $(\geq 0.6)$. The 30-day mortality was $33.3 \%(11 / 33)$ in the high IG count group and 5.4\% (2/37) in the low IG count $(<0.6)$ group $(p=0.004)$. The ICH volume was higher in the high IG count group ( $51.87 \pm 10.85$ vs. $28.91 \pm$ $6.47 ; p=0.028)$. Compared with the low IG count group, the WBC count ( $12.70 \pm 4.15$ vs. $9.21 \pm 2.80 ; p<0.001)$, the neutrophil count ( $9.80 \pm 3.72$ vs. $6.59 \pm 2.76 ; p<0.001)$, NLR $(9.41 \pm$ 6.88 vs. $5.54 \pm 5.14 ; p=0.003)$, and CRP $(48.81 \pm 10.01$ vs. $26.91 \pm 8.36 ; p=0.010$ ) were higher in the high IG count group (-Table 2). 
Table 1 Clinical and demographic characteristics of the study population

\begin{tabular}{|c|c|c|c|c|}
\hline Variables & $\begin{array}{l}\text { Total } \\
(n=70)\end{array}$ & $\begin{array}{l}\text { Good outcome } \\
(n=57)\end{array}$ & $\begin{array}{l}\text { Poor outcome } \\
(n=13)\end{array}$ & $p$-Value \\
\hline Age (years) & $68.04 \pm 13.08$ & $66.98 \pm 13.30$ & $72.69 \pm 11.34$ & 0.155 \\
\hline Male gender $n(\%)$ & $40(57.1)$ & $33(82.9)$ & $7(53.8)$ & 0.514 \\
\hline \multicolumn{5}{|l|}{ Clinical history } \\
\hline Hypertension & $49(70)$ & $39(68.4)$ & $10(76.9)$ & 0.406 \\
\hline Diabetes mellitus & $20(28.6)$ & $15(26.3)$ & $5(38.5)$ & 0.498 \\
\hline Hyperlipidemia & $15(21.4)$ & $13(22.8)$ & $2(15.4)$ & 0.720 \\
\hline Atrial fibrillation & $8(11.4)$ & $7(12.3)$ & $1(7.7)$ & 0.539 \\
\hline Coronary artery disease & $23(32.9)$ & $18(31.6)$ & $5(38.5)$ & 0.746 \\
\hline Prior stroke/TIA & $9(13)$ & $7(12.3)$ & $2(16.7)$ & 0.650 \\
\hline \multicolumn{5}{|l|}{ Vital signs at ED presentation } \\
\hline $\begin{array}{l}\text { Systolic blood pressure } \\
(\mathrm{mm} \mathrm{Hg})\end{array}$ & $165.17 \pm 36.63$ & $168.17 \pm 31.54$ & $149.00 \pm 58.46$ & 0.581 \\
\hline $\begin{array}{l}\text { Diastolic blood pressure } \\
(\mathrm{mm} \mathrm{Hg})\end{array}$ & $94.31 \pm 21.39$ & $96.06 \pm 18.70$ & $84.90 \pm 32.04$ & 0.550 \\
\hline Heart rate (BPM) & $63.31 \pm 14.56$ & $65.11 \pm 12.79$ & $53.60 \pm 19.95$ & 0.135 \\
\hline GCS & $11(3-15)$ & $12(3-15)$ & $9(3-15)$ & 0.003 \\
\hline \multicolumn{5}{|l|}{ Prestroke medications } \\
\hline Antiplatelet agents & $35(50)$ & $29(50.9)$ & $6(46.2)$ & 0.500 \\
\hline Oral anticoagulants & $8(11.4)$ & $7(12.3)$ & $1(7.7)$ & 0.539 \\
\hline Statins & $15(21.7)$ & $13(23.2)$ & $2(15.4)$ & 0.718 \\
\hline Antihypertensive drugs & $43(61.4)$ & $33(57.9)$ & $10(76.9)$ & 0.344 \\
\hline \multicolumn{5}{|l|}{ Brain imaging ICH parameters } \\
\hline Volume (mL) & $39.73 \pm 6.26$ & $31.24 \pm 5.64$ & $76.98 \pm 20.49$ & 0.005 \\
\hline \multicolumn{5}{|l|}{ Location } \\
\hline Lobar & $38(54.2)$ & $29(50.9)$ & $9(69.2)$ & \multirow[t]{3}{*}{0.028} \\
\hline Basal ganglia & $30(42.8)$ & $27(47.4)$ & $3(23.1)$ & \\
\hline Cerebellum & $2(3)$ & $1(1.7)$ & $1(7.7)$ & \\
\hline Presence of IVH & $11(15.7)$ & $6(10.5)$ & $5(38.5)$ & 0.025 \\
\hline \multicolumn{5}{|l|}{ Laboratory features } \\
\hline White blood cell $\left(10^{3} / \mathrm{mL}\right)$ & $10.97 \pm 4.04$ & $10.39 \pm 3.78$ & $13.50 \pm 4.34$ & 0.020 \\
\hline Neutrophil $\left(10^{3} / \mathrm{mL}\right)$ & $8.22 \pm 3.77$ & $7.72 \pm 3.67$ & $10.42 \pm 3.51$ & 0.011 \\
\hline Lymphocyte (103/mL) & $1.76 \pm 1.33$ & $1.71 \pm 1.11$ & $1.95 \pm 0.57$ & 0.520 \\
\hline Hemoglobin $(\mathrm{g} / \mathrm{L})$ & $12.60 \pm 2.36$ & $12.40 \pm 2.12$ & $13.46 \pm 3.16$ & 0.304 \\
\hline Platelet (103/mL) & $257.35 \pm 150.17$ & $242.36 \pm 90.47$ & $323.07 \pm 81.11$ & 0.556 \\
\hline NLR & $6.91 \pm 5.86$ & $6.41 \pm 5.82$ & $9.11 \pm 5.76$ & 0.005 \\
\hline PLR & $195.94 \pm 17.21$ & $184.56 \pm 18.21$ & $245.82 \pm 46.80$ & 0.141 \\
\hline CRP & $37.38 \pm 6.63$ & $29.26 \pm 12.18$ & $39.13 \pm 7.63$ & 0.806 \\
\hline IG & $0.76 \pm 0.08$ & $0.65 \pm 0.18$ & $1.24 \pm 0.66$ & 0.001 \\
\hline Surgery & $4(5.7)$ & $2(3.5)$ & $2(15.4)$ & 0.154 \\
\hline
\end{tabular}

Abbreviations: BPM, beats per minute; CRP, C-reactive protein; ED, emergency department; GCS, Glasgow coma scale; ICH, intracerebral hemorrhage; IG, immature granulocyte; IVH, intraventricular hemorrhage; NLR, neutrophil-lymphocyte ratio; PLR, platelet/lymphocyte ratio; TIA, transient ischemic attack.

We performed the logistic regression analysis with the variables of age ( $\geq 65$ years vs. $<65$ years), IVH (yes vs. no), ICH volume ( $\geq 30 \mathrm{~mL}$ vs. $<30 \mathrm{~mL}$ ), NLR (from high to low), and the counts of WBC and IG. These variables were selected based on studies in the literature reporting the factors associated with survival in ICH patients. ${ }^{3}$ In the regression analysis, we found out that a high IG count was significantly related to the 30-day mortality with the odds ratio (OR) of 5.157 (95\% $\mathrm{CI}=0.914-29.087, p=0.029)$. The other related factors were the presence of IVH (OR 5.801, 95\% CI $=1.024-32.873$ ), ICH 
Table 2 Clinical characteristics of population with IG $<0.6$ and IG $\geq 0.6$

\begin{tabular}{|c|c|c|c|}
\hline Variables & $\begin{array}{l}\text { Low-IG group } \\
(<0.6, n=37)\end{array}$ & $\begin{array}{l}\text { High-IG group } \\
(\geq 0.6, n=33)\end{array}$ & $p$-Value \\
\hline Age (years) & $66.18 \pm 13.33$ & $70.12 \pm 12.66$ & 0.189 \\
\hline Male gender $n(\%)$ & $23(62.2)$ & $17(51.1)$ & 0.469 \\
\hline \multicolumn{4}{|l|}{ Clinical history } \\
\hline Hypertension & $27(73)$ & $22(66.7)$ & 0.609 \\
\hline Diabetes mellitus & $9(24.3)$ & $11(33.3)$ & 0.438 \\
\hline Hyperlipidemia & 7 (18.9) & $8(24.2)$ & 0.771 \\
\hline Atrial fibrillation & $5(13.5)$ & $3(9.1)$ & 0.714 \\
\hline Coronary artery disease & $14(37.8)$ & $9(27.3)$ & 0.447 \\
\hline Prior stroke/TIA & $4(11.1)$ & $5(15.2)$ & 0.728 \\
\hline \multicolumn{4}{|l|}{ Vital signs at ED presentation } \\
\hline Systolic blood pressure (mm Hg) & $170.67 \pm 33.47$ & $158.11 \pm 39.82$ & 0.381 \\
\hline Diastolic blood pressure (mm Hg) & $96.44 \pm 19.64$ & $91.57 \pm 23.58$ & 0.743 \\
\hline Heart rate (bpm) & $64.83 \pm 12.97$ & $61.36 \pm 16.43$ & 0.669 \\
\hline GCS & $12(3-15)$ & $10(3-15)$ & 0.041 \\
\hline \multicolumn{4}{|l|}{ Prestroke medications } \\
\hline Antiplatelet agents & $19(51.4)$ & $16(48.5)$ & 0.500 \\
\hline Oral anticoagulants & $5(13.5)$ & $3(9.1)$ & 0.714 \\
\hline Statins & $7(18.9)$ & $8(25)$ & 0.572 \\
\hline Antihypertensive drugs & $22(59.5)$ & $21(63.6)$ & 0.808 \\
\hline \multicolumn{4}{|l|}{ Brain imaging $\mathrm{ICH}$ parameters } \\
\hline Volume $(\mathrm{mL})$ & $28.91 \pm 6.47$ & $51.87 \pm 10.85$ & 0.028 \\
\hline \multicolumn{4}{|l|}{ Location } \\
\hline Lobar & $13(35.1)$ & $25(75.7)$ & \multirow[t]{3}{*}{0.007} \\
\hline Basal ganglia & $23(62.1)$ & $7(21.3)$ & \\
\hline Cerebellum & $1(2,8)$ & $1(3)$ & \\
\hline Presence of IVH & $4(11.1)$ & $7(21.3)$ & 0.327 \\
\hline \multicolumn{4}{|l|}{ Laboratory features } \\
\hline White blood cell $\left(10^{3} / \mathrm{mL}\right)$ & $9.21 \pm 2.80$ & $12.70 \pm 4.15$ & $<0.001$ \\
\hline Neutrophil $\left(10^{3} / \mathrm{mL}\right)$ & $6.59 \pm 2.76$ & $9.80 \pm 3.72$ & $<0.001$ \\
\hline Lymphocyte $\left(10^{3} / \mathrm{mL}\right)$ & $1.69 \pm 0.71$ & $1.83 \pm 0.32$ & 0.205 \\
\hline Hemoglobin $(\mathrm{g} / \mathrm{L})$ & $12.62 \pm 2.07$ & $12.68 \pm 2.64$ & 0.469 \\
\hline Platelet $(103 / \mathrm{mL})$ & $229.75 \pm 81.13$ & $293.84 \pm 198.94$ & 0.210 \\
\hline NLR & $5.54 \pm 5.14$ & $9.41 \pm 6.88$ & 0.003 \\
\hline PLR & $159.25 \pm 101.06$ & $242.65 \pm 173.63$ & 0.038 \\
\hline CRP & $26.91 \pm 8.36$ & $48.81 \pm 10.01$ & 0.010 \\
\hline Surgery & $2(5.4)$ & $2(6.1)$ & 0.648 \\
\hline 30-day mortality (n [\%]) & $2(5.4)$ & $11(33.3)$ & 0.004 \\
\hline
\end{tabular}

Abbreviations: CRP, C-reactive protein; ED, emergency department; GCS, Glasgow coma scale; ICH, intracerebral hemorrhage; IG, immature granulocyte; IVH, intraventricular hemorrhage; NLR, neutrophil-lymphocyte ratio; PLR, platelet/lymphocyte ratio; TIA, transient ischemic attack.

volume (OR 1.012, 95\% CI= 1.003-1.025), and NLR (OR 0.197, 95\% CI $=0.039-0.989)$ ( - Table 3). Furthermore, the effectiveness of IG in predicting the 30-day mortality was calculated by plotting ROC curves ( - Fig. 1). At a cutoff point of 0.6 for the IG count, AUC was 0.789 (0.665-0.914); sensitivity was $84.6 \%$, and specificity was $74.2 \%$.

\section{Discussion}

The most important new findings of our study are the significantly high IG count in nonsurvivors of ICH compared with the survivors and the high mortality rates in the patients with an IG count of 0.6 or higher. Our results have shown 
that the IG count can provide a risk classification to predict the 30-day outcome in ICH patients.

Studies have shown that inflammation takes an important part in the pathophysiology of $\mathrm{ICH}$. With the start of bleeding, many complex inflammatory reactions are activated including the activation of microglia. ${ }^{13,14}$ The activation of microglia leads to the infiltration of various circulating immune cells, mainly macrophages and $\mathrm{T}$ cells, resulting in the release of inflammatory cytokines, chemokines, and free radicals. These, along with cell death products, further activate the resident and migrating lymphocytes, leading to increased infiltration of lymphocytes and a continuous cycle of inflammatory responses. There is increasing evidence that this inflammatory response contributes to the formation of edema through increased blood-brain barrier (BBB) permeability around the hematoma, which exacerbates the mass effect and increases the cell death process through secondary ischemia, and more inflammatory, surrounding brain tissue. ${ }^{14,15}$ Therefore, it has recently become the center of

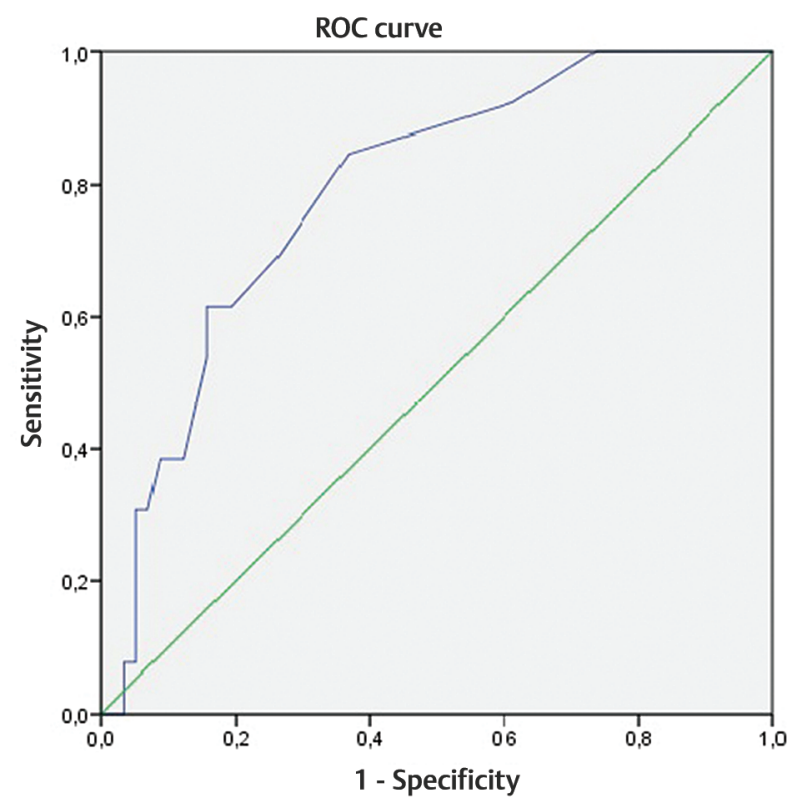

Fig. 1 Receiver operating characteristic (ROC) curve analysis of immature granulocyte (IG) in the discrimination between mortality. attention that new parameters of inflammation can predict the prognosis in ICH patients.

Previous studies have associated the WBC and neutrophil count, NLR, PLR, and other inflammatory parameters with poor prognosis in ICH patients. ${ }^{16-18}$ The study by Wang et $\mathrm{al}^{4}$ highlighted that the 30-day outcome was poor in $\mathrm{ICH}$ patients, with an NLR of 7.35 and above. Another study by Luo et al $^{19}$ demonstrated that a high NLR was a new risk factor for mortality and morbidity in ICH in patients with type 2 diabetes mellitus. A study conducted by Zhang et $\mathrm{al}^{20}$ reported that a high PLR value in ICH patients found in the emergency department before admission to the intensive care unit was a significant predictor for the short-term neurological outcome but not for the long-term survival. Another recent study showed that a high WBC and neutrophil count, a high NLR, and a low lymphocyte count could be predictive of poor survival after ICH. ${ }^{21}$ In alignment with the literature, we found out in our study that a high WBC and a high neutrophil count and a high NLR were related to the 30-day survival in ICH patients, but we could not find a significant relationship of the lymphocyte count and PLR to the survival.

Studies have indicated that the 30-day survival in ICH patients is associated with a high ICH volume, low GCS scores, and surgical procedures. ${ }^{22,23}$ Studies report that surgery is required in patients with a hematoma volume of $30 \mathrm{~mL}$ and higher but survival is not good in those patients. ${ }^{24}$ In the decision of surgery, it is essential that the clinician make his/her own best decision, and the primary efficacy in this decision is the location of the hematoma, age, neurological condition, hematoma volume, comorbid condition, and the drugs used. Generally, applied in our hospital, the classic treatment is conservative follow-up. Mainly, antiedema is the general supportive treatment, which is carried out by applying the therapy and keeping the systemic arterial blood pressure at the desired level. Dexamethasone is mostly preferred in antiedema treatment. The dose is adjusted by considering the dynamics of each case. In cases where dexamethasone is contraindicated, mannitol $20 \%$ solution is used. In antihypertensive treatment, if the patient is not comatose, peroral dietetics, calcium antagonists, or $\beta$ blockers are used. Parenteral diuretics are preferred in patients with extremely impaired consciousness. Nitroprusside is also used in resistant hypertensive cases. In our study, we found out that

Table 3 Logistic regression analysis of risk factors affected UGIB 30-day mortality

\begin{tabular}{|l|l|l|l|l|}
\hline \multirow{2}{*}{ Variables } & \multicolumn{2}{|c|}{ Univariate analysis } & \multicolumn{2}{c|}{ Multivariate analysis } \\
\cline { 2 - 5 } & OR $(95 \% \mathrm{CI})$ & $p$-Value & OR $(95 \% \mathrm{CI})$ & $p$-Value \\
\hline Age & $1.036(0.986-1.089)$ & 0.160 & & 0.047 \\
\hline Presence of IVH & $5.312(1.308-21.574)$ & 0.020 & $5.801(1.024-32.873)$ & 0.014 \\
\hline ICH volume & $1.013(1.003-1.024)$ & 0.013 & $1.012(1.003-1.025)$ & 0.862 \\
\hline White blood cell $(103 / \mathrm{mL})$ & $1.196(1.031-1.387)$ & 0.018 & $0.862(0.191-3.891)$ & 0.048 \\
\hline NLR & $1.121(1.021-1.230)$ & 0.017 & $0.197(0.039-0.989)$ & 0.029 \\
\hline IG & $2.613(1.115-6.124)$ & 0.027 & $5.157(0.914-29.087)$ & \\
\hline
\end{tabular}

Abbreviations: $\mathrm{Cl}$, confidence interval; CRP, C-reactive protein; ICH, intracerebral hemorrhage; IG\%, immature granulocyte percentage; IGC, immature granulocyte count; IVH, intraventricular hemorrhage; NLR, neutrophil-to-lymphocyte ratio; OR, odds ratio; UGIB, upper gastrointestinal bleeding. 
low GCS scores and a high ICH volume significantly affected the 30-day survival.

The IG count is a new parameter that gives the number of promyelocytes, myelocytes, and metamyelocytes in peripheral blood. A low IG count can be detected by automated blood analyzers more reliably compared with conventional counting under a light microscope. ${ }^{25}$ Being not a normal component in peripheral blood, there is IG increase in number in several conditions including trauma, necrosis, and acute transplant rejection..$^{25}$ In these conditions, an increase in the IG count indicates an increase in the number of neutrophils that escape the bone marrow. Moreover, some people, especially old individuals, newborns, and myelosuppressed patients, may not display an increased neutrophil count and may even have neutropenia in other conditions such as sepsis. An increase in the IG count in such conditions may help in showing the presence of acute infection. Furthermore, studies have found out that the granulocyte count is significantly high in many inflammatory conditions such as acute appendicitis, liver abscesses, and infective complications after cardiac surgery. ${ }^{26,27}$ Huang et $\mathrm{al}^{8}$ reported that a high IG count can predict the risk of acute respiratory distress syndrome (ARDS) early in patients with acute pancreatitis. Another study by Lipinski et $\mathrm{al}^{28}$ showed that an IG count of 0.6 and above had high specificity and sensitivity in demonstrating acute pancreatitis complications. Senthilnayagam et $\mathrm{al}^{7}$ reported that IG can be used potential markers for bacterial infection. In this study, IG > 3 showed sepsis with high sensitivity and specificity. To the best of our knowledge, no studies are available in the literature examining the relationship between the IG count and ICH. In our study, we found out that the IG count was significantly high in patients with poor prognosis for the 30-day survival. The patients with an IG count of 0.6 and above had a significantly poor prognosis for the 30-day survival.

Our study had some limitations. First, it is a single-center and retrospective study conducted with somewhat a small number of patients. Second, the IG count was obtained only at the time of admission. The lack of the availability of serial counts is the most important limitation of our study. Another limitation is the lack of the availability of the levels of the tumor necrosis factor and interleukin-6, which we could not test in the emergency department. Our results might have been more productive if the IG count had been found to be related to such inflammatory parameters. Multicentre prospective studies with a larger patient population are needed to better interpret the findings of our study.

\section{Conclusions}

An IG count can be obtained from a simple full blood count, easy to apply, and does not require extra costs. The IG count is used as a marker to predict the 30-day prognosis.

\section{Ethical Approval}

This study was approved by Institutional Ethics Committee, Antalya, Turkey. All the patients or caregivers gave their written informed consent for research, which was conducted in accordance with the Helsinki Declaration.

\section{Authors' Contributions}

All the authors have a substantial contribution in the study design, data interpretation, and writing and reviewing the manuscript.

\section{Funding}

None.

\section{Conflict of Interest}

None declared.

\section{References}

1 Zhang J, Cai L, Song Y, et al. Prognostic role of neutrophil lymphocyte ratio in patients with spontaneous intracerebral hemorrhage. Oncotarget 2017;8(44):77752-77760

2 Lattanzi S, Silvestrini M, Provinciali L. Elevated blood pressure in the acute phase of stroke and the role of Angiotensin receptor blockers. Int J Hypertens 2013;2013:941783

3 Mracsko E, Veltkamp R. Neuroinflammation after intracerebral hemorrhage. Front Cell Neurosci 2014;8:388

4 Wang F, Hu S, Ding Y, et al. Neutrophil-to-lymphocyte ratio and 30-day mortality in patients with acute intracerebral hemorrhage. J Stroke Cerebrovasc Dis 2016;25(1):182-187

5 Morotti A, Phuah CL, Anderson CD, et al. Leukocyte count and intracerebral hemorrhage expansion. Stroke 2016;47(6): 1473-1478

6 Bolayir A, Cigdem B, Gokce SF, et al. The effect of eosinopenia on mortality in patients with intracerebral hemorrhage. J Stroke Cerebrovasc Dis 2017;26(10):2248-2255

7 Senthilnayagam B, Kumar T, Sukumaran J, M J, Rao K R. Automated measurement of immature granulocytes: performance characteristics and utility in routine clinical practice. Pathol Res Int 2012;2012:483670

8 Huang Y, Xiao J, Cai T, et al. Immature granulocytes: A novel biomarker of acute respiratory distress syndrome in patients with acute pancreatitis. J Crit Care 2019;50:303-308

9 Yoon SH, Shin $\mathrm{H}$, Lee $\mathrm{KH}$, et al. Predictive factors for bacteremia in febrile infants with urinary tract infection. Sci Rep 2020;10(1):4469

$10 \mathrm{Kim} \mathrm{OH}$, Cha YS, Hwang SO, et al. The use of delta neutrophil index and myeloperoxidase index for predicting acute complicated appendicitis in children. PLoS One 2016;11(2):e0148799

11 Huttner HB, Steiner T, Hartmann M, et al. Comparison of $\mathrm{ABC} / 2$ estimation technique to computer-assisted planimetric analysis in warfarin-related intracerebral parenchymal hemorrhage. Stroke 2006;37(2):404-408

12 Kaya İ, Cingöz İD, Uzunoğlu İ, Kızmazoğlu C, Aydın HE, Yüceer N. Predictors of Mortality in Patients with Surgically Treated Spontaneous Intracranial Hemorrhage. Turk J Neurol 2019;25: 214-217

13 Yao Y, Tsirka SE. The CCL2-CCR2 system affects the progression and clearance of intracerebral hemorrhage. Glia 2012;60(6): 908-918

14 Bian L, Zhang J, Wang M, Keep RF, Xi G, Hua Y. Intracerebral hemorrhage-induced brain injury in rats: the role of extracellular peroxiredoxin 2. Transl Stroke Res 2020;11(2):288-295

15 Tschoe C, Bushnell CD, Duncan PW, Alexander-Miller MA, Wolfe SQ. Neuroinflammation after intracerebral hemorrhage and potential therapeutic targets. J Stroke 2020;22(1):29-46

16 Adeoye O, Walsh K, Woo JG, et al. Peripheral monocyte count is associated with case fatality after intracerebral hemorrhage. J Stroke Cerebrovasc Dis 2014;23(2):e107-e111 
17 Sun W, Peacock A, Becker J, Phillips-Bute B, Laskowitz DT, James ML. Correlation of leukocytosis with early neurological deterioration following supratentorial intracerebral hemorrhage. J Clin Neurosci 2012;19(8):1096-1100

18 Wang F, Xu F, Quan Y, et al. Early increase of neutrophilto-lymphocyte ratio predicts 30-day mortality in patients with spontaneous intracerebral hemorrhage. CNS Neurosci Ther 2019;25(1):30-35

19 Luo P, Li R, Yu S, et al. The relationship between neutrophilto-lymphocyte ratio and intracerebral hemorrhage in type 2 diabetes mellitus.J Stroke Cerebrovasc Dis 2017;26(5):930-937

20 Zhang W, Shen Y. Platelet-to-lymphocyte ratio as a new predictive index of neurological outcomes in patients with acute intracranial hemorrhage: a retrospective study. Med Sci Monit 2018;24:4413-4420

21 Lattanzi S, Cagnetti C, Provinciali L, Silvestrini M. Neutrophilto-lymphocyte ratio predicts the outcome of acute intracerebral hemorrhage. Stroke 2016;47(6):1654-1657

22 Li Q, Zhang G, Xiong X, et al. Black hole sign: novel imaging marker that predicts hematoma growth in patients with intracerebral hemorrhage. Stroke 2016;47(7):1777-1781
23 Lattanzi S, Cagnetti C, Provinciali L, Silvestrini M. Neutrophilto-lymphocyte ratio and neurological deterioration following acute cerebral hemorrhage. Oncotarget 2017;8(34):5748957494

24 Cho DY, Chen CC, Lee HC, Lee WY, Lin HL. Glasgow Coma Scale and hematoma volume as criteria for treatment of putaminal and thalamic intracerebral hemorrhage. Surg Neurol 2008;70(6):628-633

25 Bruegel M, Fiedler G, Matthes G, Thiery J. Reference values for immature granulocytes in healthy blood donors generated on the Sysmex XE-2100 automated hematology analyser. Sysmex J Int 2004;14(1):5-7

26 Ünal Y. A new and early marker in the diagnosis of acute complicated appendicitis: immature granulocytes. Ulus Travma Acil Cerrahi Derg 2018;24(5):434-439

27 Daix T, Guérin E, Tavernier E, et al. Immature granulocytes: a risk factor of infection after cardiac surgery. Cytometry B Clin Cytom 2018;94(6):887-894

28 Lipiński M, Rydzewska G. Immature granulocytes predict severe acute pancreatitis independently of systemic inflammatory response syndrome. Prz Gastroenterol 2017;12(2):140-144 